Proceedings

\title{
Sensing of Furfural by Molecularly Imprinted Polymers on Plasmonic and Electrochemical Platforms ${ }^{\dagger}$
}

\author{
Maria Pesavento ${ }^{1, *}$, Nunzio Cennamo ${ }^{2}$, Giancarla Alberti ${ }^{~}$, Simone Marchetti ${ }^{1}$ and Luigi Zeni ${ }^{2}$ \\ 1 Department of Chemistry, University of Pavia, Via Taramelli n.12, 27100 Pavia, Italy; \\ giancarla.alberti@unipv.it (G.A.); simone.marchetti01@universitadipavia.it (S.M.) \\ 2 Department of Engineering, University of Campania Luigi Vanvitelli, Via Roma n.29, 81031 Aversa, Italy; \\ nunzio.cennamo@unicampania.it (N.C.); luigi.zeni@unicampania.it (L.Z.) \\ * Correspondence: maria.pesavento@unipv.it \\ + Presented at the 7th International Symposium on Sensor Science, Napoli, Italy, 9-11 May 2019. \\ Published: 18 December 2019
}

\begin{abstract}
The goal of this work is to test the possibility of selective detection of furfural (2-FAL) in aqueous solutions, with a molecularly imprinted polymer (MIP) receptor exploiting two different transduction methods, for food safety applications. In particular, sensors with electrochemical and surface plasmon resonance (SPR) transduction are considered. Two concentration ranges could be investigated by the different sensing approaches since the detectable concentration level depends on the sensitivity of the detection technique employed. The determination of 2-FAL at different concentration levels in the aqueous medium of interest, as for example beverages, is becoming a very crucial task not only for the relevance of furanic compounds in affecting the flavor but also for their possible toxic and carcinogenic effects on the human beings. For these reasons, their determination by a fast, easy and low-cost method is of interest. The sensor methods here proposed appears to be particularly suitable, since, although together and not individually, they allow the determination in a wide concentration range.
\end{abstract}

Keywords: furfural (2-FAL); molecularly imprinted polymers (MIPs); electrochemical sensors; surface plasmon resonance (SPR); plastic optical fibers (POFs); optical-chemical sensors

\section{Introduction}

Chemical sensors are based on the strict integration of a receptor with an instrument able to generate a signal upon the combination of the receptor with the substrate. Both the characteristics of the binding reaction, in particular the affinity constant, and those of the transductor, for example the sensitivity, are of overwhelming relevance for determining the performance of a sensor.

In the present work, we examined a solid synthetical receptor obtained by non-covalent molecular imprinting of 2-furaldheide (2-FAL) connected with two different transduction methods. Solids of this kind, obtained by the molecular imprinting methods, have been widely demonstrated to be advantageous with respect to the biological receptors, in particular in the field of sensing, in terms of reproducibility, fast and low-cost development, stability in time and possibility of application in non-physiological conditions. However, as well known, heterogeneous binding sites are formed in MIPs synthesized by a non-covalent bulk procedure [1], which results in different binding affinities and allows to perform measurements at different concentration levels. The detectable concentration level, in turn, depends on the sensitivity of the detection technique employed. The heterogeneity of MIP may diminish the performance in some analytical application, but it can be interesting in the case of sensing since it could make the coupling with different transduction methods possible. 
In the present investigation, the possibility of using the same MIP, most probably heterogeneous, in connection with different transduction methods is examined. In particular, sensors with electrochemical and SPR transduction are considered. In both these devices the MIP can be easily deposited over the transductor (chip). From previous experience it is expected that different concentration ranges of 2-FAL in aqueous matrices can be measured by the two methods, if sites with the appropriate affinity are present in the receptor considered.

\section{Sensing Platforms}

Electrochemical sensors are chemically modified electrodes (CME), with their surface functionalized by opportune species capable of specifically interacting with the desired analyte are fixed. A signal (a potential, a current or an impedance variation) is generated by the transductor upon the interaction sensor/analyte, which can be used to determine the concentration of the analyte, or the characteristics of the combination reaction. The devices here considered are created by depositing an MIP layer over a screen printed electrochemical cell (SPC) in a simple way. This method makes it possible to study MIP reactions exactly in the form of a thin layer as it is used in the sensing devices.

Various MIP-based electrochemical sensors have been developed in the last years by our research groups for the determination of different small molecules by potentiometric $[2,3]$ and voltammetric [4] transduction.

Voltammetric sensors based on screen printed cell (SPC) are particularly convenient for characterization, because of their low cost and very easy preparation. The SPC-MIP sensor, considered in the present investigation, is composed of a graphite ink working and auxiliary electrode, and a silver ink quasi-reference electrode obtained by the screen printed technique; the MIP layer is deposed over the whole cell. In the sensor here proposed the signal is a peak current due to the faradic process of the substrate, i.e., 2-FAL, since it is electroactive. In this work the signal is the peak current $\left(i_{p}\right)$ obtained by square wave voltammetry (SWV), which is directly proportional to the analyte concentration in the polymeric layer near the working electrode.

A completely different transduction principle too was exploited to study the binding properties of the considered MIP for 2-FAL, i.e., a sensor based on the optical phenomenon of the SPR. The MIPSPR platform used in the present investigation is different from the most usual ones, with Kretschmann configuration, since it is based on a D-shaped plastic optical fiber (POF), with a planar surface obtained by erasing the cladding and partially the core, over which a multilayer structure was realized as previously described [5,6]. It is suitable for investigating the binding properties of the MIP since the signal obtained, i.e., the variation of the resonance wavelength (Wav shift) is a consequence of the binding of the substrate with MIP. Actually, it is due to the variation of the refractive index of the MIP layer on the gold film [6].

\section{Experimental Results}

Different results have been obtained with the two different characterization techniques. For a general comparison the dose-response curves of the same MIP with both different methods are reported in Figure 1. It is seen that the electrochemical sensor covers a concentration range different from that of the MIP-SPR sensor, which responds in a lower concentration range, only partially overlapping with the other. The MIP-SPR response is obtained for 2-FAL concentrations much lower than that by the electrochemical sensing, in agreement with its expected higher sensitivity. Since different 2-FAL concentrations are present in different beverages, by these two methods we could investigate several food matrices. 


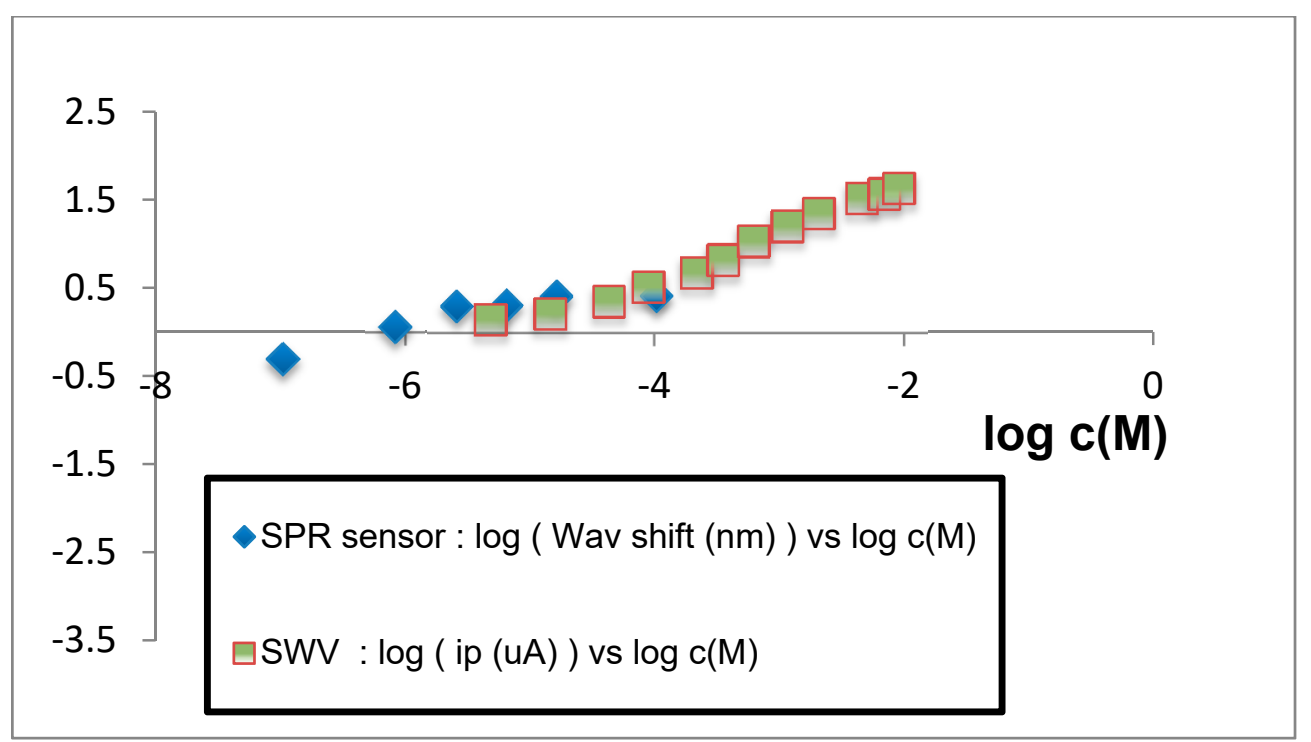

Figure 1. Responses of MIP receptor for 2-FAL in aqueous solution by SPR-POF and electrochemical sensors (in $\log -\log$ scales).

\section{Conclusions}

As shown in Figure 1, when the electrochemical approach is used, it was possible to detect 2FAL in aqueous solution at concentrations from about $3 \times 10^{-6} \mathrm{M}$ to $1 \times 10^{-2} \mathrm{M}$. The corresponding detection range in the case of the SPR sensor is from $1 \times 10^{-7} \mathrm{M}$ to $1 \times 10^{-4} \mathrm{M}$. The SPR sensor sensitivity is very low at concentration higher than about $1 \times 10^{-5} \mathrm{M}$. It is possible that heterogeneous binding sites are involved even in the case of a single transduction method. It is interesting to notice that the electrochemical sensor shows an LOD of about $6 \times 10^{-6} \mathrm{M}$ for 2-FAL in water, which is almost one order of magnitude higher than that obtained by the SPR sensor. For example, this LOD presented by the SPR based sensor is suitable for the determination of 2-FAL in wine, since this is usually the concentration level in this food matrix.

Author Contributions: M.P., N.C., L.Z., and G.A.: investigation, conceptualization, methodology, validation, formal analysis, and writing. S.M.: investigation, and writing.

Funding: This research was funded by “VALERE” program of the University of Campania Luigi Vanvitelli (Italy).

Conflicts of Interest: The authors declare no conflicts of interest.

\section{References}

1. Umpleby, R.J.; Baxter, S.C.; Chen, Y.; Shah, R.N.; Shimizu, K.D. Characterization of molecularly imprinted polymers with the Langmuir-Freundlich isotherm. Anal. Chem. 2001, 73, 4584-4591.

2. Pesavento, M.; D’Agostino, G.; Biesuz, R.; Alberti, G.; Profumo, A. Ion Selective Electrode for Dopamine Based on a Molecularly Imprinted Polymer. Electroanalysis 2011, 24, 813-824.

3. D’Agostino, G.; Alberti, G.; Biesuz, R.; Pesavento, M. Potentiometric sensor for atrazine based on a molecular imprinted membrane. Biosens. Bioelectron. 2006, 15, 145-152.

4. Pesavento, M.; D’Agostino, G.; Alberti, G.; Biesuz, R.; Merli, D. Voltammetric platform for detection of 2,4,6-trinitrotoluene based on a molecularly imprinted polymer. Anal. Bioanal. Chem. 2013, 405, 3559-3570.

5. Cennamo, N.; Massarotti, D.; Conte, L.; Zeni, L. Low Cost Sensors Based on SPR in a Plastic Optical Fiber for Biosensor Implementation. Sensors 2011, 11, 11752-11760.

6. Cennamo, N.; De Maria, L.; Chemelli, C.; Profumo, A.; Zeni, L.; Pesavento, M. Markers detection in transformer oil by plasmonic chemical sensor system based on POF and MIPs. IEEE Sens. J. 2016, 16, 7663-7670. 